\title{
Leguminosae no Pantanal de Barão de Melgaço, Mato Grosso, Brasil
}

\author{
Rosilene Rodrigues Silva ${ }^{1,2}$ \\ ${ }^{1}$ Vale Complexo Corumbá, Gerência de Meio Ambiente, CP 221, CEP 79301-970, Corumbá, MS, Brasil \\ ${ }^{2}$ Autor para correspondência: Rosilene Rodrigues Silva, e-mail: rosilener663@gmail.com
}

SILVA, R.R. Leguminosae in the Pantanal of Barão de Melgaço, Mato Grosso, Brazil. Biota Neotrop. 11(4): http://www.biotaneotropica.org.br/v11n4/en/abstract?short-communication+bn00711042011

Abstract: The Brazilian Pantanal is composed of eleven sub-regions, one of them the Pantanal of Barão de Melgaço, located northeast of the Quaternary sedimentary basin. Considering that Leguminosae is identified as one of most abundant families in the Pantanal region, this study aimed to present a list of taxa of Leguminosae taxa occurring in the Pantanal of Barão de Melgaço, municipality of Barão de Melgaço, Mato Grosso state. Information about the habit and vegetation type are provided for all the species. A total of 40 táxons were recorded and its belong to 23 genera. Most species are tree and occur primarily in riparian vegetation. This study shows, among the species reported, two new records for the Mato Grosso state, that are: Aeschynomene evenia C. Wright ex Sauvalle and Pterocarpus villosus (Benth.) Benth.

Keywords: floristic, Fabaceae, riparian vegetation, Aeschynomene, Pterocarpus.

SILVA, R.R. Leguminosae no Pantanal de Barão de Melgaço, Mato Grosso, Brasil. Biota Neotrop. 11(4): http://www.biotaneotropica.org.br/v11n4/pt/abstract?short-communication+bn00711042011

Resumo: O Pantanal brasileiro é constituído por onze sub-regiões, sendo uma delas a do Pantanal de Barão de Melgaço, localizado ao nordeste da bacia sedimentar quaternária. Considerando que Leguminosae é apontada como uma das famílias mais abundantes na região do Pantanal, este trabalho teve por objetivo apresentar uma lista dos táxons de Leguminosae ocorrentes no Pantanal de Barão de Melgaço, município de Barão de Melgaço, estado do Mato Grosso. Para as espécies são fornecidas informações sobre o hábito e tipo de vegetação. Um total de 40 táxons foi registrado, pertencendo a 23 gêneros. A maioria das espécies são árvores e ocorrem essencialmente nas formações ciliares. Este estudo aponta, dentre as espécies encontradas, dois novos registros para o estado do Mato Grosso, que são: Aeschynomene evenia C. Wright ex Sauvalle e Pterocarpus villosus (Benth.) Benth. Palavras-chave: florística, Fabaceae, vegetação ciliar, Aeschynomene, Pterocarpus. 


\section{Introdução}

Leguminosae ou Fabaceae é uma das três maiores famílias de angiospermas, com 19.327 espécies e 727 gêneros arranjados em 36 tribos (Lewis et al 2005). Está dividida em três subfamílias, Caesalpinioideae, Mimosoideae e Papilionoideae, morfologicamente distintas entre si, e está distribuída por todos os ecossistemas terrestres (Polhill et al. 1981, Lewis et al. 2005). A ocorrência da família no Brasil é bastante significativa, representada por 210 gêneros e 2694 espécies (Lima et al. 2010), ocupando todos os biomas brasileiros (Lima 2000, Souza \& Lorenzi 2008). Em termos econômicos, as sementes, legumes, folhas, raízes e flores de muitas de suas espécies são fontes de alimentos ricos em proteínas para homens e animais em quase todas as partes do mundo (Lewis \& Owen 1989).

O Pantanal brasileiro, distribuído entre os estados de Mato Grosso e Mato Grosso do Sul, foi dividido em 11 sub-regiões, a saber: Cáceres, Poconé, Barão de Melgaço, Paraguai, Paiaguás, Nhecolândia, Abobral, Aquidauana, Miranda, Nabileque e Porto Murtinho (Silva \& Abdon 1998). O Pantanal de Barão de Melgaço, localizado no setor nordeste da bacia sedimentar quaternária, ocupa $18.167 \mathrm{~km}^{2}$ de área do Pantanal (Silva \& Abdon 1998). Compreende uma área suscetível a alagamentos periódicos de sua planície, por extravasamento das águas do Rio Cuiabá (Borges et al. 2000), o qual nasce na serra azul, município de Rosário Oeste, e apresenta uma bacia de drenagem com vales bastante íngremes no alto do curso, que vão se alargando e formando extensas áreas alagáveis até a foz, onde desemboca no Rio Paraguai (Carvalho 1986). Ao longo do percurso deste rio, nas áreas consideradas mais baixas, ocorre a formação de baías que se conectam com o mesmo, abastecendo-o no período de cheia com o extravasamento de suas águas, como no caso das baías de Chacororé, Sá-Mariana, Porto de Fora e Acurizal, no pantanal de Barão de Melgaço. A heterogeneidade espacial de solos nesta região está relacionada com a diversidade de feições geomórficas, as quais estão adicionadas aos tipos de inundação, gerando uma grande diversidade de microhabitats, como cordilheiras, paleodiques, murunduns, baías, corixos e vazantes que apresentam inundações anuais ou permanentes (Beirigo 2008).

Com relação à flora matogrossense, Leguminosae é apontada como uma das famílias mais expressiva (Dubs 1998). Também, nos diferentes estudos florísticos já realizados em áreas do Pantanal brasileiro, é reconhecida como uma das famílias de maior importância na composição de diversas formações vegetacionais, apresentando elevado número de espécies (Nunes da Cunha 1990, Pott \& Pott 1994, 1999, Schessl 1999, Duarte 2007, Lima Júnior 2007, Silva 2010). Pelo exposto, o presente trabalho tem como objetivo ampliar o conhecimento deste grupo taxonômico, fornecendo uma listagem dos táxons de Leguminosae ocorrentes no município de Barão de Melgaço, Pantanal de Barão de Melgaço, incluindo informações sobre hábito e tipo de vegetação de ocorrência.

\section{Material e Métodos}

As coletas ao acaso foram realizadas no Pantanal de Barão de Melgaço, município de Barão de Melgaço, altitudes entre 100 e 150 m, nas comunidades de Estirão Comprido e Porto São João, Baías Acurizal e Chacororé, Posto Espírito Santo e RPPN Sesc Pantanal. A sub-região de Barão de Melgaço, de acordo com Silva \& Abdon (1998), participa com 13,15\% da área total do Pantanal, com aproximdamente $18.500 \mathrm{~km}^{2}$. Agrega os municípios de Itiquira, Barão de Melgaço e Santo Antônio do Leverger, todos no estado do Mato Grosso. O clima da região é Aw-tropical úmido, segundo a classificação de Köppen (1948). A maioria dos solos ocorre em ambientes com predomínio de processos redoximórficos, onde os elementos com Fé e Mn são sucestíveis as alterações em sua mobilidade no solo, sendo os principais processos pedogenéticos a gleização e plintização (Beirigo 2008). A vegetação encontrada na área de estudo está constituída por cambarazal, campo de murundus, vegetação secundária (campo de pastagem), campo sazonalmente inundável, campo sujo, campo úmido, cerrado, cerrado inundável, cerradão, floresta estacional decidual, mata ciliar e mata inundável (terminologia de acordo com Pott \& Pott 1994, Silva et al. 2000, Duarte 2007).

$\mathrm{Na}$ amostragem florística foram considerados todos os hábitos, desde ervas até árvores. O material em fase reprodutiva foi incorporado na coleção do Herbário UFMT (acrônimo de acordo com Holmgren \& Holmgren (1998)).

A lista das espécies e categorias infra-específicas foi elaborada a partir de sete expedições realizadas entre os períodos de julho de 2007 a dezembro de 2008, com duração de três dias cada, durante as estações seca e chuvosa. Além disso, foram examinados materiais provenientes da sub-região de Barão de Melgaço depositados no Herbário UFMT. A terminologia utilizada na caracterização dos tipos de hábito foi baseada em Guedes-Bruni et al. (2002) e, para a definição da vegetação e seus equivalentes regionais, na de Pott \& Pott (1994), Silva et al. (2000) e Duarte (2007). O termo vegetação secundária refere-se às áreas perturbadas, modificadas pela ação antrópica. A classificação de Leguminosae em subfamílias seguiu a proposta por Lewis et al. (2005). A identificação dos táxons foi feita por meio de obras originais e/ou revisões e descrições encontradas na literatura e da comparação com material depositado no Herbário RB (Holmgren \& Holmgren 1998). Para a padronização dos nomes dos autores das espécies, foi consultada a página do IPNI-The International Pant Names Index, com base em Brummitt \& Powell (1992).

\section{Resultados e Discussão}

O levantamento florístico no município de Barão de Melgaço resultou na identificação de 40 táxons específicos e subespecíficos de Leguminosae, distribuídos em 23 gêneros e três subfamílias (Tabela 1). Para a subfamília Caesalpinioideae foram registradas 16 espécies distribuídas em sete gêneros. Bauhinia é o mais expressivo (6 spp.), seguido por Senna (4 spp.), Hymenaea (2 spp.). e Cassia, Copaifera, Phanera e Tachigali, com uma espécie cada. O resultado encontrado para Bauhinia corrobora o verificado por Lima Júnior et al. (2007) para espécies arbustivo-arbóreas na mesma área, no qual o gênero sobressaiu pelo maior número de espécies em relação aos demais. Para a subfamília Mimosoideae foram registradas 14 espécies distribuídas em nove gêneros. Inga é o gênero melhor representado (4 spp., 1 subesp.), seguido por Mimosa (3 spp.), Zygia (2 spp.) e Albizia, Anadenanthera, Calliandra, Enterolobium, Samanea e Senegalia, com uma única espécie cada. Para a subfamília Papilionoideae foram registradas 10 espécies distribuídas em oito gêneros. Aeschynomene e Pterocarpus são os mais bem representados, com duas espécies cada, seguidos por Andira, Ateleia, Dipteryx, Indigofera, Leptolobium e Swartzia, com apenas uma espécie cada. As subfamílias com maior número de representantes foram Caesalpinioideae e Mimosoideae, no entanto, dentre as espécies de Papilionoideae encontradas, são confirmados dois novos registros para o estado do Mato Grosso, que são: Aeschynomene evenia C. Wright ex Sauvalle e Pterocarpus villosus (Mart. ex Benth.) Benth. Estas espécies não foram citadas em estudos de flora do estado de Mato Grosso (Dubs 1998); também não constam na listagem preliminar de fanerógamas para a flora do Pantanal (Pott \& Pott 1999), o que demonstra que o Pantanal como um todo deve ser floristicamente melhor estudado. Aeschynomene evenia não é endêmica do Brasil e, no Brasil, é encontrada em região da Amazônia, Caatinga, Cerrado, Mata Atlântica e Pantanal (Lima \& Oliveira 2010), ocorrendo nos 
Tabela 1. Lista dos táxons de Leguminosae ocorrentes no município de Barão de Melgaço, Pantanal de Barão de Melgaço, Mato Grosso, Brasil, depositados no Herbário UFMT, Cuiabá.

Table 1. List of taxa of Leguminosae collected in the Barão de Melgaço municipality, Pantanal de Barão de Melgaço, Mato Grosso, Brazil, deposited at the UFMT herbarium, Cuiabá.

\begin{tabular}{|c|c|c|}
\hline Táxon & Habito/vegetação & $\mathrm{N}^{0}$ registro \\
\hline \multicolumn{3}{|l|}{ CAESALPINIOIDEAE } \\
\hline Bauhinia cupulata Benth. & Arbusto/11 & UFMT 29055 \\
\hline Bauhinia curvula Benth. & Arbusto/5 & UFMT 37772 \\
\hline Bauhinia mollis (Bong.) D. Dietr. & Arbusto/7 & UFMT 37859 \\
\hline Bauhinia pentandra (Bong.) Vogel ex Steud. & Arbusto/10 & UFMT 37690 \\
\hline Bauhinia pulchella Benth. & Arbusto/8 & UFMT 35894 \\
\hline Bauhinia ungulata $\mathrm{L}$. & Arbusto/11 & UFMT 21115 \\
\hline Cassia grandis L. f. & Árvore/11 & UFMT 38806 \\
\hline Copaifera langsdorffii Desf. & Árvore /12 & UFMT 19563 \\
\hline Hymenaea courbaril $\mathrm{L}$. & Árvore /10 & UFMT 38809 \\
\hline Hymenaea stigonocarpa Mart. ex Hayne & Árvore/7 & UFMT 37888 \\
\hline Phanera glabra (Jacq.) Vaz & Cipó/10 & UFMT 39106 \\
\hline Senna alata (L.) Roxb. & Arbusto/11 & UFMT 38800 \\
\hline Senna obtusifolia (L.) H.S. Irwin \& Barneby & Subarbusto/6 & UFMT 39107 \\
\hline Senna occidentalis (L.) Link & Subarbusto/3 & UFMT 37957 \\
\hline Senna pendula (Humb. \& Bonpl. ex Willd.) H.S. Irwin \& Barneby & Arbusto/3 & UFMT 37959 \\
\hline Tachigali aurea Tul. & Árvore/8 & UFMT 27208 \\
\hline \multicolumn{3}{|l|}{ MIMOSOIDEAE } \\
\hline Albizia inundata (Mart.) Barneby \& J.W. Grimes & Árvore/4 & UFMT 36680 \\
\hline Anadenanthera colubrina var. cebil (Griseb.) Altschul & Árvore/10 & UFMT 38798 \\
\hline Calliandra parviflora Benth. & Arbusto/10 & UFMT 37784 \\
\hline Enterolobium contortisiliquum (Vell.) Morong & Árvore/10 & UFMT 38807 \\
\hline Inga grandiflora Ducke & Árvore/1 & UFMT 35773 \\
\hline Inga laurina (Sw.) Willd. & Árvore/11 & UFMT 36679 \\
\hline Inga striata Benth. & Árvore/1 & UFMT 35748 \\
\hline Inga vera subsp. affinis (DC.) T.D. Penn. & Árvore/11 & UFMT 36692 \\
\hline Mimosa bimucronata (DC.) Kuntze & Árvore/11 & UFMT 27798 \\
\hline Mimosa debilis Humb. \& Bonpl. ex Willd. & Arbusto/11 & UFMT 27797 \\
\hline Mimosa pellita Humb. \& Bonpl. ex Willd. & Arbusto/11 & UFMT 37953 \\
\hline Samanea tubulosa (Benth.) Barneby \& J. W. Grimes & Árvore/7 & UFMT 36693 \\
\hline Senegalia polyphylla (DC.) Britton \& Rose & Árvore/10 & UFMT 31141 \\
\hline Zygia inaequalis (Willd.) Pittier & Árvore/12 & UFMT 27199 \\
\hline Zygia latifolia var. comunis Barneby \& J.W. Grimes & Árvore/11 & UFMT 20783 \\
\hline \multicolumn{3}{|l|}{ PAPILIONOIDEAE } \\
\hline Aeschynomene evenia $\mathrm{C}$. Wright \& Sauvalle & Subarbusto/5 & UFMT 16520 \\
\hline Aeschynomene fluminensis Vell. & Subarbusto/11 & UFMT 38808 \\
\hline Andira inermis (W. Wright) DC. & Árvore/2 & UFMT 37893 \\
\hline Ateleia guaraya Herzog & Árvore/7 & UFMT 36690 \\
\hline Dipteryx alata Vogel & Árvore/2 & UFMT 25562 \\
\hline Indigofera lespedezioides Kunth & Subarbusto/5 & UFMT 28031 \\
\hline Leptolobium nitens Vogel & Subarbusto/1 & UFMT 35743 \\
\hline Pterocarpus santalinoides L’Hér. ex DC. & Árvore/12 & UFMT 26910 \\
\hline Pterocarpus villosus (Mart. ex Benth.) Benth. & Árvore/11 & UFMT 29078 \\
\hline Swartzia jorori Harms & Árvore/11 & UFMT 38801 \\
\hline
\end{tabular}

1: cambarazal; 2: campo de murundus; 3: campo de pastagem; 4: campo sazonalmente inundável; 5: campo sujo; 6: campo úmido; 7: cerrado; 8: cerrado inundável; 9: cerradão; 10: floresta estacional decidual; 11: mata ciliar; 12: mata inundável:

1: cambarazal, 2: murundus campo, 3: pasture campo, 4: seasonally flooded campo; 5: dirty campo, 6: wet campo, 7: cerrado, 8: flooded cerrado, 9: cerradão, 10: seasonal deciduous forest, 11: riparian vegetation, 12: flooded forest. 
estados do Pará, Maranhão, Ceará, Bahia, Minas Gerais, Rio de Janeiro e Paraná. Por outro lado, P. villosus é endêmica do Brasil, restrita à região da Caatinga, e citada apenas para os estados da Bahia e Piauí (Lima 2010).

Nos diversos ambientes da área de estudo, as Leguminosae ocorreram como árvores (23 spp.-57\%), arbustos (11 spp.-27\%), subarbustos (6 spp.-15\%) ou cipós (1 sp.-2,5\%). Espécies herbáceas não foram encontradas. Ao comparar as informações de hábito e vegetação, verifica-se que a metade das leguminosas arbóreas predominou nas matas ciliares, fitofisionomia que apresentou a maior diversidade de Leguminosae (13 ssp., 1 subesp.). Este padrão expressivo das espécies arbóreas de Leguminosae em matas ciliares é semelhante ao encontrado por Segalla (2008), em estudo realizado na Estação Ecológica Serra das Araras, no estado do Mato Grosso. Com relação às espécies arbustivas de Leguminosae, observa-se que estas ocuparam diferentes ambientes como, mata ciliar, cerrado, cerrado inundável, cerradão, floresta decídua e campo de pastagem. Todavia, as espécies subarbustivas ocorreram na vegetação secundária (campo de pastagem), campo sujo, campo úmido e mata ciliar. Das espécies subarbustivas com ocorrência no campo de pastagem, Senna occidentalis (L.) Link, conhecida popularmente na região como "fedegoso", é considerada ecologicamente como indicadora de grande perturbação dos habitats (Pott \& Pott 1994). O único cipó encontrado foi Phanera glabra (Jacq.) Vaz conhecida popularmente na região do Pantanal por "tripa-de-galinha", com ocorrência na vegetação de floresta decídua.

$\mathrm{Na}$ fitofisionomia de floresta estacional decidual foi encontrado o segundo maior número de representantes de Leguminosae (6 spp., 1 var.). Observa-se que dos seis táxons, quatro são arbóreos, entre eles: Hymenaea courbaril L. (Caesalpinioideae), Anadenanthera colubrina var. cebil (Griseb.) Altschul, Enterolobium contortisiliquum (Vell.) Morong e Senegalia polyphylla (DC.) Britton \& Rose (Mimosoideae). A ocorrência de A. colubrina e $S$. polyphylla foi confirmada em estudos realizados em outras áreas de floresta seca do Brasil (Salis et al. 2004, Silva 2006, Siqueira 2007).

Entre as espécies relacionadas para a região, Bauhinia curvula Benth. (Leguminosae-Caesalpinioideae), Andira inermis (W. Wright) DC. e $P$. villosus (Leguminosae-Papilionoideae) são as únicas consideradas endêmicas para o Brasil (Lima 2010, Vaz 2010, Pennington 2010).

Os táxons registrados neste estudo não são exclusivos do bioma Pantanal, apresentando distribuição em outros domínios fitogeográficos, como a Amazônia, Caatinga, Cerrado e Mata Atlântica (Lima et al. 2010). Isso ocorre, segundo Pott et al. (2009), porque o Pantanal é ocupado por plantas que migram de biomas circundantes. Observa-se, entretanto, poucas espécies de ampla distribuição na área de estudo, especificamente, duas de Caesalpinioideae: Hymenaea courbaril L. e Senna occidentalis (L.) Link, uma de Mimosoideae: Senegalia polyphylla (DC.) Britton \& Rose, e duas de Papilionoideae: Aeschynomene evenia e Aeschynomene fluminensis Vell. (Lima \& Oliveira 2010, Morim \& Barros 2010, Ribeiro 2010, Souza \& Bortoluzzi 2010). As demais espécies possuem uma distribuição mais centralizada. Todas as espécies aqui citadas já haviam sido relacionadas nos estudos para o Pantanal (Nunes da Cunha 1990, Pott \& Pott 1994, 1999, Silva 2010), com exceção de Leptolobium nitens Vogel. Este estudo preenche parcialmente as lacunas de conhecimento sobre as Leguminosae, uma vez que não abrangeu todos os municípios da sub-região de Barão de Melgaço.

\section{Agradecimentos}

Ao Conselho Nacional de Desenvolvimento Científico e Tecnológico $(\mathrm{CNPq})$ pela bolsa concedida à autora, Processo individual no 350125/2007-4. Ao técnico do Herbário UFMT Sr. José Edvaldo da Silva pelo auxílio nas coletas do material botânico.
À especialista Laura Cristina Pires pela confirmação das espécies de Aeschynomene. Á Dra. Rafaela Campostrini Forzza pela solicitude prestada durante a visita ao Herbário RB. Este trabalho foi financiado pela Fundação de Amparo à Pesquisa no Estado do Mato Grosso (FAPEMAT), Processo no 1.024/2006.

\section{Referências Bibliográficas}

BEIRIGO, R.M. 2008. Sistema pedológico Planosolo-Plintossolo no Pantanal de Barão de Melgaço-MT. Dissertação de Mestrado, Universidade de São Paulo, Piracicaba.

BORGES, J.R.P., CHAUDHRY, F.H. \& FERREIRA FILHO, P. 2000. Um estudo sobre a percepção de ribeirinhos da Bacia do Alto Paraguai a respeito da questão de justiça e eqüidadade em aproveitamento hídrico: o caso da hidrovia Paraguai-Paraná. In III Simpósio sobre recursos naturais e sócio-econômicos do Pantanal: os desafios do novo milênio. Corumbá. http://www.cpap.embrapa.br/agencia/congresso/Socio/BORGES-036.pdf (último acesso em 20/01/2010).

BRUMMITT, R.K. \& POWELL, C.E. 1992. Authors of plants names. Royal Botanic Gardens, Kew.

CARVALHO, N.O. 1986. Hidrologia da bacia do Alto Paraguai. In I Simpósio Sobre Recursos Naturais e Sócio-econômicos do Pantanal. Embrapa, Brasília, p.43-49.

DUARTE, T.G. 2007. Florística, Fitossociologia e Relações Solo-vegetação em Floresta Estacional Decidual em Barão de Melgaço, Pantanal de Mato Grosso. Tese de Doutorado, Universidade Federal de Viçosa, Viçosa.

DUBS, B. 1998. Prodromus Florae Matogrossensis. The Botany of MatoGrosso, Betrona-Verlag, Küsnacht. Part 1. Checklist of Angiosperm, part 2. Types from MatoGrosso.

GUEDES-BRUNI, R.R., MORIM, M.P., LIMA, H.C. \& SYLVESTRE, L.S. 2002. Inventário florístico. In Manual metodológico para estudos botânicos na Mata Atlântica (L.S. Sylvestre \& M.M.T. Rosa, ed.). Universidade Federal Rural do Rio de Janeiro, Seropédica, p.24-49.

HOLMGREN, P.K. \& HOLMGREN, N.H. 1998. Onwards (continuously updated). Index Herbariorum: a global directory of public herbaria and associated staff. New York Botanical Garden, New York. http://sweetgun. nybg.org/ih/ (últimoacessoem12/01/2010).

KÖPPEN, W. 1948. Climatologia. Fondo de Cultura, Buenos Aires.

LEWIS, G.P. \& OWEN, P.E. 1989. Legumes of the Ilha de Maracá. Royal Botanic Gardens, Kew.

LEWIS, G.P., SCHIRE, B.D., MACKINDER, B.A. \& LOCK, J.M. 2005. Legumes of the World. Royal Botanic Gardens, Kew.

LIMA, H.C. 2000. Leguminosas arbóreas da mata atlântica: uma análise da riqueza, padrões de distribuição geográfica e similaridades florísticas em remanescentes florestais no estado do Rio de Janeiro. Tese de doutorado, Universidade Federal do Rio de Janeiro, Rio de Janeiro.

LIMA, H.C. 2010. Pterocarpus. In Listade espécies da flora do Brasil (Jardim Botânico do Rio de Janeiro). Jardim Botânico do Rio de Janeiro. http:// floradobrasil.jbrj.gov.br/2010/FB019003 (último acesso em 07/01/2011).

LIMA, H.C., QUEIROZ, L.P., MORIM, M.P., SOUZA, V.C., DUTRA, V.F., BORTOLUZZI, R.L.C., IGANCI, J.R.V., FORTUNATO, R.H., VAZ, A.M.S.F., SOUZA, E.R., FILARDI, F.L.R., VALLS, J.F.M., GARCIA, F.C.P., FERNANDES, J.M., SILVA, R.C.V. M., FORTUNA-PEREZ, A.P., MANSANO, V.F., MIOTTO, S.T.S., TOZZI, A.M.G.A., MEIRELES, J.E., LIMA, L.C.P., OLIVEIRA, M.L.A.A., FLORES, A.S., TORKE, B.M., PINTO, R.B., LEWIS, G.P., BARROS, M.J.F., RIBEIRO, R.D., SCHÜTZ, R., PENNINGTON, T., KLITGAARD, B.B., RANDO, J.G., SCALON, V.R., CARDOSO, D.B.O.S., COSTA, L.C., SILVA, M.J., MOURA, T.M., BARROS, L.A.V., SILVA, M.C.R., QUEIROZ, R.T., SARTORI, A.L.B. \& CAMARGO, R. 2010. Fabaceae. In Lista de espécies da flora do Brasil (Jardim Botânico do Rio de Janeiro). Jardim Botânico do Rio de Janeiro, Rio de Janeiro. http://floradobrasil.jbrj.gov.br/2010/FB000115 (último acesso em 07/01/2011).

LIMA JÚNIOR, G.A. 2007. Flora e estrutura do estrato arbóreo e relações solovegetação de cerradão em Barão de Melgaço, Pantanal de Mato Grosso, Brasil. Dissertação de Mestrado, Universidade Federal de Viçosa, Viçosa. 
LIMA JÚNIOR, G.A., SOARES, T.S., NUNES DA CUNHA, C. \& FERREIRA, H. 2007. Estudo florístico das espécies arbustivas e arbóreas nas fitofisionomias da RPPN Sesc Pantanal, Mato Grosso, Brasil. In VIII Congresso de Ecologia do Brasil. Sociedade de Ecologia do Brasil, Caxambu. http://www.seb-ecologia.org.br/viiiceb/pdf/631.pdf (último acesso em 18/01/2010).

LIMA, L.C.P. \& OLIVEIRA, M.L.A.A. 2010. Aeschynomene. In Listade espécies da flora do Brasil (Jardim Botânico do Rio de Janeiro). Jardim Botânico do Rio de Janeiro, Rio de Janeiro. http://floradobrasil.jbrj.gov. br/2010/FB082595 (último acesso em 07/01/2011).

MORIM, M.P. \& BARROS, M.J.F. 2010. Senegalia. In Listade espécies da flora do Brasil (Jardim Botânico do Rio de Janeiro). Jardim Botânico do Rio de Janeiro, Rio de Janeiro. http://floradobrasil.jbrj.gov.br/2010/ FB101015 (último acesso em 7/01/2011).

NUNES DA CUNHA, C. 1990. Estudo florístico e fitossociológico das principais formações arbóreas do Pantanal de Poconé-MT. Dissertação de Mestrado, Universidade Estadual de Campinas, Campinas.

PENNINGTON, T. 2010. Andira. In Listade espécies da flora do Brasil (Jardim Botânico do Rio de Janeiro). Jardim Botânico do Rio de Janeiro, Rio de Janeiro. http://floradobrasil.jbrj.gov.br/2010/FB022787 (último acesso em 07/01/2011)

POLHILL, R.M., RAVEN, P.H. \& STIRTON, C.H. 1981.Evolution and systematics of the Leguminosae. In Advances in Legumes Systematics 1 (R.M. Polhill \& P.H. Raven, ed.). Royal Botanic Gardens, Kew, p.1-26.

POTT, A \& POTT, V.J. 1994. Plantas do Pantanal. Embrapa, Brasília.

POTT, A. \& POTT, V.J. 1999. Flora do Pantanal-Listagem atual de fanerógamas. In II Simpósio sobre Recursos Naturais e Sócio-Econômicos do Pantanal. Manejo e Conservação, Embrapa Pantanal, Corumbá, p.298-325.

POTT, A., POTT, V.J. \& DAMASCENO JUNIOR, G.A. 2009. Fitogeografia do Pantanal. In III Congresso Latino Americano de Ecologia-CLAE, IX Congresso de Ecologia do Brasil-CEB. São Lourenço, p.1-4.

RIBEIRO, R.D. 2010. Hymenaea. In Listade espécies da flora do Brasil (Jardim Botânico do Rio de Janeiro). Jardim Botânico do Rio de Janeiro, Rio de Janeiro. http://floradobrasil.jbrj.gov.br/2010/FB022972 (último acesso em 07/01/2011).
SCHESSL, M. 1999. Floristic composition and structure of floodplain vegetation in northern Pantanal of MatoGrosso, Brasil. Phyton 39(2):303-336.

SALIS, S.M., SILVA, M.P., MATTOS, P.P., SILVA, J.S.V., POTT, JV.J. \& POTT, A. 2004. Fitossociologia de remanescentes de floresta estacional decidual em Corumbá, Estado do Mato Grosso do Sul, Brasil. Rev. Bras. Bot. 27(4):671-684. http://dx.doi.org/10.1590/S010084042004000400008

SEGALLA, R. 2008. Florística e estrutura fitossociológica de duas matas ciliares em diferentes estádios sucessionais na Estação Ecológica Serra das Araras, Mato Grosso. Dissertação de Mestrado, Universidade Federal de Mato Grosso, Cuiabá.

SILVA, J.S.V. \& ABDON, M.M. 1998. Delimitação do pantanal brasileiro e suas sub-regiões. Pesq. Agropec. Bras. 33:1703-1711.

SILVA, M.P., MAURO, R., MOURÃO, G. \& COUTINHO, M. 2000. Distribuição e quantificação de classes de vegetação do Pantanal através de levantamento aéreo. Rev. Bras. Bot. 23(2):143-152.

SILVA, R.R. 2006. Leguminosae no Planalto Residual do Urucum, oeste do Pantanal do Mato Grosso do Sul, Brasil: inventário, taxonomia e similaridade florística. Tese de Doutorado, Universidade Estadual de Campinas, Campinas.

SILVA, R.R. 2010. Leguminosae do município de Poconé, Pantanal de Poconé, Mato Grosso, Brasil. Biota Neotr. 10(4):1-7.

SIQUEIRA, A.S. 2007. Florística, fitossociologia e caracteres edáficos de duas florestas estacionais deciduais no triângulo mineiro. Dissertação de Mestrado, Universidade Federal de Uberlândia, Minas Gerais.

SOUZA, V.C. \& LORENZI, H. 2008. Botânica sistemática: guia ilustrado para identificação das famílias de fanerógamas nativas e exóticas no Brasil, baseado em APG II. Instituto Plantarum, Nova Odessa.

SOUZA, V.C. \& BORTOLUZZI, R.L.C. 2010. Senna. In Listade espécies da flora do Brasil (Jardim Botânico do Rio de Janeiro). Jardim Botânico do Rio de Janeiro, Rio de Janeiro. http://floradobrasil.jbrj.gov.br/2010/ FB023162 (último acesso em 07/01/2011).

VAZ, A.M.S.F. 2010. Bauhinia. In Lista de espécies da flora do Brasil (Jardim Botânico do Rio de Janeiro). Jardim Botânico do Rio de Janeiro, Rio de Janeiro. http://floradobrasil.jbrj.gov.br/2010/FB027767(último acesso em 07/01/2011). 